\title{
Características de la arena sílica de Alvarado, Veracruz, México, como material para barreras reactivas permeables, para la remediación de acuíferos contaminados con lixiviados de basureros no controlados
}

(Characteristics of Silica Sand from Alvarado, Veracruz, Mexico as material for Permeable Reactive Barriers for the Remediation of Aquifers contaminated with leachates from uncontrolled landfills)

Manuel Alberto Susunaga-Miranda', Bertha María Estévez-Garrido ${ }^{2}$, Rodrigo Manuel Susunaga-Estévez ${ }^{3}$

\section{Resumen}

En este artículo se caracteriza la arena sílica de las costas del municipio de Alvarado, Veracruz, México, y se compara sus propiedades con los materiales usados en las barreras reactivas permeables, para permitir su uso, como una solución de remediación de acuíferos contaminados con lixiviados de basureros no controlados. Esto se logra mediante análisis granulométrico, determinación de humedad y concentración de sílice y fierro, con lo cual se obtienen partículas con tamaño de 0.707 mm, humedad relativa de $0.52 \%$ y concentración de $\mathrm{SiO}_{2}$ y de $\mathrm{Fe}_{2} \mathrm{O}_{3} 87.38 \%$ y $2.72 \%$ respectivamente, lo que, al comparar con materiales reactivos en uso, cumple con los criterios para ser usado como barrera para la remediación de acuíferos con lixiviados.

\section{Palabras clave}

Arena Sílica, Barreras Reactivas Permeables, Basureros, Remoción de contaminantes, granulometría, materiales reactivos

\begin{abstract}
This article characterizes the silica sand from the coasts of the municipality of Alvarado, Veracruz, Mexico and compares its properties with the materials used for the Permeable Reactive Barriers and that allow its use as a solution for the remediation of aquifers contaminated with leachate from uncontrolled landfills, by granulometric analysis, determination of humidity and concentration of Silica and Iron, obtaining particles with a diameter of $0.707 \mathrm{~mm}$, relative humidity of $0.52 \%$ and concentration of $\mathrm{SiO}_{2}$ and $\mathrm{Fe}_{2} \mathrm{O}_{3} 87.38 \%$ and $2.72 \%$ respectively, which comparing with reactive materials in use meets the criteria to be used as a Barrier for the Remediation of Aquifers contaminated with leachates
\end{abstract}

\section{Keywords}

Silica Sand, Permeable Reactive Barriers, Dumpsites, contamination removal, particle size, reactive materials

\section{Introducción}

El incremento de la población junto con la agricultura y las actividades industriales, han generado un aumento en la demanda de agua para consumo humano y, ante la escasez de la misma, a

\footnotetext{
1 Tecnológico Nacional de México/Instituto Tecnológico de Veracruz. Veracruz, México. [manuel.sm@veracruz.tecnm.mx, https://orcid.org/0000-0002-5595-0914]

2 Tecnológico Nacional de México/Instituto Tecnológico de Veracruz. Veracruz, México. [bertha.eg@everacruz.tecnm.mx, https://orcid.org/0000-0002-8543-3520]

3 Tecnológico Nacional de México/Instituto Tecnológico de Veracruz. Veracruz, México. [L19020931@veracruz.tecnm.mx, https://orcid.org/0000-0002-6570-9307]
} 
nivel superficial en algunos lugares o debido a su contaminación, la preservación de este recurso en el subsuelo tiene una gran importancia. Si bien el agua subterránea representa solo un pequeño porcentaje de los recursos hídricos totales en la Tierra, su contribución es vital, teniendo en cuenta que hasta dos mil millones de personas dependen directamente de los acuíferos para el agua potable y el 40 \% de los alimentos del mundo es producido por la agricultura de regadío, que depende en gran medida de aguas subterráneas (Thiruvenkatachari et al. 2008).

Una de las principales fuentes de contaminación de los acuíferos subterráneos está relacionado con la disposición de residuos sólidos urbanos (RSU), los cuales generan un líquido lixiviado, que se forma por una parte por la percolación del agua de lluvia y de la infiltración del nivel freático, y por la otra, por la descomposición anaeróbica de los residuos (Susunaga y Estévez, 2018), cuya composición depende de la naturaleza de los desechos y de los procesos químicos y bioquímicos de transformación de la materia y sus características fundamentales cambian con el paso del tiempo (Naveen et al., 2017, con componentes altamente peligrosos, como compuestos aromáticos, halogenados, fenoles, pesticidas, metales pesados y amoniaco.

Cuando los residuos sólidos terminan en un basurero no controlado, se genera el lixiviado, cuya cantidad se ve afectada principalmente por las precipitaciones, la evapotranspiración, la escorrentía superficial, la infiltración de agua subterránea y el grado de compactación de los desechos (Miao et al., 2019), lo cual migra con el agua por efecto de la gravedad, afectando la calidad de las aguas superficiales y de los acuíferos subterráneos (Teta \& Hikwa, 2017), y eventualmente, llegarían por medio de las descargas de los ríos al mar, lo que pone en peligro la biota acuática (Susunaga y Estévez, 2018).

A medida que los lixiviados se acumulan en el acuífero, la extensión de la contaminación se ve afectada por factores ambientales, como la precipitación, la tasa de contaminación, la geología del sitio, su permeabilidad y régimen de flujo de aguas subterráneas (Han et al., 2016). El tratamiento de las aguas subterráneas contaminadas y la recuperación de los acuíferos suele ser una de las tareas de complejidad elevada y alto costo económico, que depende entre otras cosas de las características hidrogeológicas del emplazamiento, del tipo de contaminante y del grado de afectación de la zona (Chimenos \& Navarro, 2002).

En los sitios de disposición final de los residuos sólidos urbanos ya sean rellenos sanitarios, basureros controlados o tiradero a cielo abierto (NOM-083-SEMARNAT-083, 2004), uno de los principales problemas es el control de los lixiviados, ya que esto implica mantener su flujo dentro de los mismos sitios y darles un tratamiento adecuado. Sin embargo, en el 2010, en el 15 $\%$ de estos sitios, se escapaban a diario ocasionando contaminación de fuentes de agua y suelos. Ya para el año 2011, en la región centro occidente de México (donde se incluye al estado de Veracruz), el 46 \% de los sitios de disposición final de residuos sólidos urbanos no contaban con fosas o lagunas para captar lixiviados; además, el 61 \% de estos no tenía instaladas geomembranas, por lo tanto la contaminación escurre pendiente abajo hacia los acuíferos subterráneos (Bernaché, 2012).

Las barreras reactivas permeables (BRP) constituyen una técnica de tratamiento de aguas subterráneas in situ, que consiste en la intercepción del flujo del agua subterránea para reducir o transformar los contaminantes presentes en ella (Striegel et al., 2001, Yin et al., 2017), entre los que se encuentran los metales pesados y los compuestos clorados (Maitra, 2019). La principal ventaja de las BRP sobre los métodos ex situ u otros métodos in situ de remediación de aguas subterráneas, es la naturaleza pasiva del tratamiento. Es decir, en su mayor parte, su funcionamiento no depende de mano de obra externa o insumos de energía. Una vez instalada, 
la barrera aprovecha el flujo de agua subterránea in situ para que los contaminantes entren en contacto con los medios reactivos llenados (Liu et al., 2011).

Los materiales de las BRP eliminan los contaminantes disueltos del agua subterránea, mediante la inmovilización dentro de la barrera o la transformación a productos menos nocivos, a través de mecanismos que incluyen la adsorción y precipitación, la reacción química y las reacciones biológicas (Scherer et al., 2000). Entre los materiales que han sido probados, el desarrollo de las barreras reactivas permeables se encuentran, la zeolita natural, zeolita modificada con hierro, apatita, apatita concentrada, caolín y bentonita cruda han (Vukojević et al., 2017), ceniza de carbón, caolinita, cáscara de coco (Asokbunyarat y Annachhatre 2017), pirita, hierro granular (Maitra, 2019), arcillas (Segura et al., 2015), y de algún modo la arena sílica, que se ha utilizado como parte de una mezcla con escoria y alúmina activada (McRae, 1999). Estos materiales en su mayoría tienen una alta capacidad para el intercambio de cationes, y crean una carga neta negativa mediante la sustitución de cationes de valencia inferior (por ejemplo, $\mathrm{Al}^{3+}$ ), con un catión de mayor valencia (por ejemplo, $\mathrm{Si}^{4+}$ ), dentro de la estructura mineral (Bohn, 1985). En México se ha comprobado que arcillas modificadas o activadas presentan una eficiencia de remoción de $\mathrm{Cr}+6$ hasta del 95.37 \% y que son un material eficiente para control de lixiviados de tiraderos a cielo abierto (Segura et al 2015).

Las cenizas volantes y cenizas de fondo, cuyo contenido principal son los óxidos de silicio $\left(\mathrm{SiO}_{2}\right)$, aluminio $\left(\mathrm{Al}_{2} \mathrm{O}_{3}\right)$, hierro $\left(\mathrm{Fe}_{2} \mathrm{O}_{3}\right)$ y calcio $(\mathrm{CaO}$, tienen excelentes características de adsorción, principalmente por su carga superficial y por la presencia de iones de fierro. El átomo central de silicio tiene una fuerte afinidad electrónica, lo que produce que tengan poca basicidad y como resultado, esto hace que su superficie actúe como un ácido débil, formando grupos funcionales de silanol $\left(\mathrm{SiOH}^{-3}\right)$, cuando los átomos de oxígeno superficiales reaccionan con el agua (Mohan y Gandhimathi 2009).

La arena sílica es un compuesto resultante de la combinación del Silicio con el Oxígeno. Su composición química está formada por un átomo de sílice y dos átomos de Oxígeno, formando una molécula muy estable: $\mathrm{SiO}_{2}$. se produce por trituración de piedra o arena de sílica de textura abierta, cribada a distribución de grano necesaria. La arena de sílice es producida por trituración de molienda y lavado de la sílice de alta calidad. Esta arena se utiliza como un medio granular filtrante en el tratamiento del agua potable y residual. Características físicas: arena de gránulo duro. El tamaño de grano de las partículas es variable, cribable, y principalmente de color marrón a gris. Se produce en números de malla 10 × 20 y 20 × 30. La producción nacional de sílice en 2013 fue de 2.9 millones de toneladas, 18.2 \% menor con relación al 2012. Los principales estados productores son Coahuila (35.65 \%), Veracruz (55.35 \%) y Nuevo León (6.55 \%). (Dirección general de Desarrollo Minero, 2011).

Una de las características primordiales para minimizar las limitaciones del flujo del agua subterránea, es el tamaño de las partículas, ya que un amplio rango de tamaños y muy pequeñas dimensiones podría resultar en un bloqueo entre los espacios intergranulares (Faisal, 2018).

Sin embargo, hasta el momento no se habian realizado experimentaciones con óxido de silicio $\left(\mathrm{SiO}_{2}\right)$ en forma de arena sílica, por lo que el objetivo fundamental de este trabajo es el de determinar que los componentes del material proveniente de la costa del Municipio de Alvarado, y cuentan con las características suficientes para ser empleados como barrera reactiva permeable para la remediación de la contaminación de los acuíferos subterráneos provenientes de lixiviados de basureros no controlados. 


\section{Metodología}

\section{a) El Municipio de Alvarado, Veracruz, México}

El municipio de Alvarado se localiza en la llanura costera del Golfo, en la parte central del Estado de Veracruz (figura 1); tiene una extensión territorial de $840 \mathrm{~km}^{2}$ y limita al norte con Boca del Río, al sur con Ignacio de la Llave, Acula, Tlacotalpan y Lerdo de Tejada; al Este con el Golfo de México y al Oeste con Medellín de Bravo y Tlalixcoyan, en la denominada Región del Papaloapan (Sefiplan, 2012), con predominio de ecosistema costero, con dunas, manglares y pastizales (Vázquez-lule et al. 2009).

Figura 1 Localización del Municipio de Alvarado, Veracruz, México

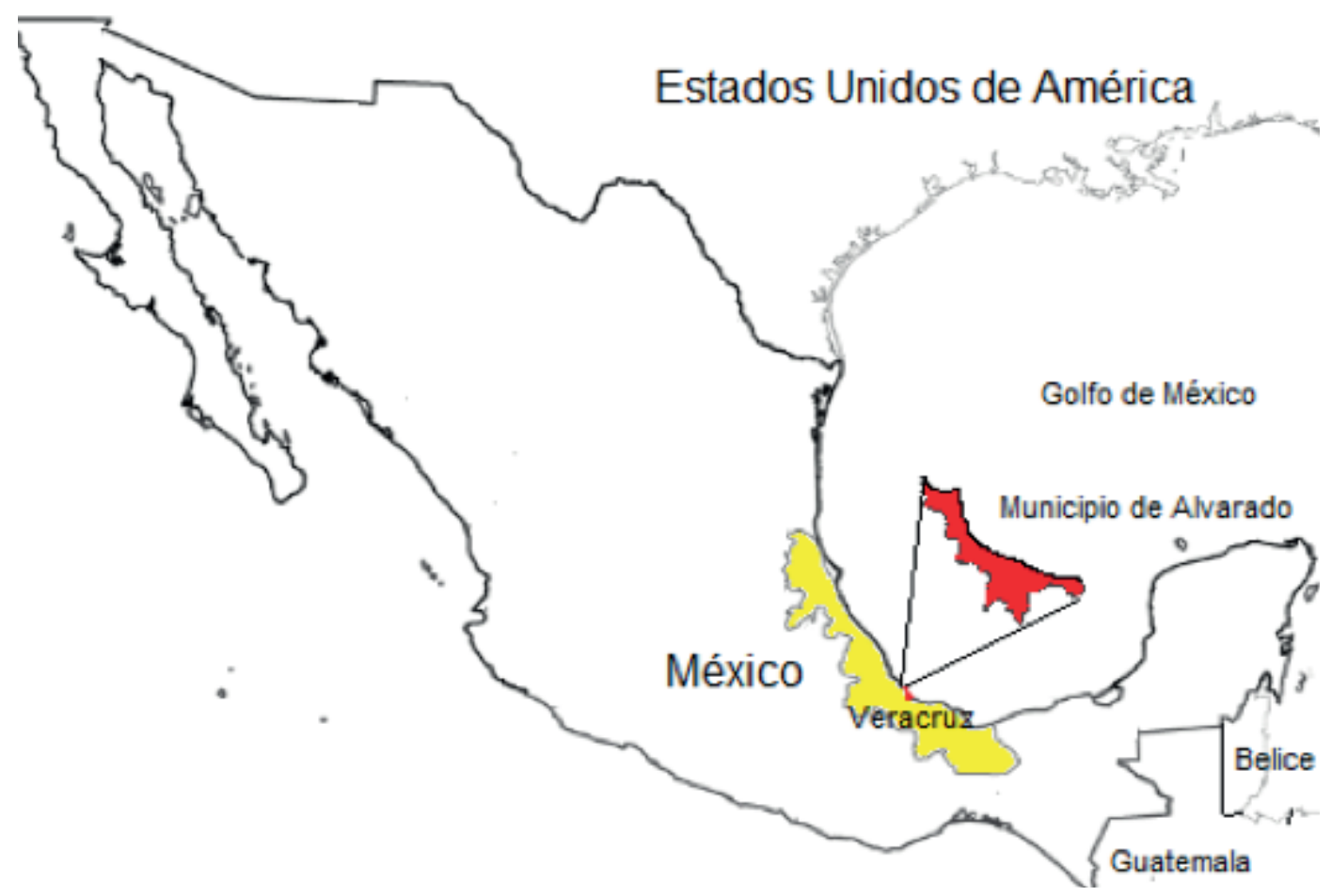

Fuente: Elaboración Propia

\section{b) Los bancos de arena sílica de la costa del Municipio de Alvarado, Veracruz}

La zona de donde se extrae la arena sílica está localizada en la costa sur del municipio de Alvarado, Veracruz, a $5.2 \mathrm{~km}$ de la desembocadura del río Papaloapan por la margen derecha (figura 2). Esta es una región de depósitos sedimentarios, producto de la interacción de los aportes fluviales, las aguas del Golfo de México y de los vientos y oleajes predominantes, con formaciones de la era cuaternaria, especialmente del pleistoceno (Díaz et al., 2018).

Los bancos de arena sílica se localizan en las coordenadas UTM GSW grado 15 (tabla 1). 
Tabla 1. Coordenadas de banco de arena sílica de Alvarado, Veracruz, México

\begin{tabular}{|c|c|c|}
\hline Vértice & $\mathbf{X}$ & $\mathbf{Y}$ \\
\hline 1 & 214137.18 & 2076871.73 \\
\hline 2 & 214105.21 & 2076734.61 \\
\hline 3 & 215186.75 & 2076214.84 \\
\hline
\end{tabular}

Para llegar a los bancos de arena sílica, desde la Ciudad de Alvarado, Veracruz, se toma la carretera Federal 180 en dirección a Lerdo de Tejada, por una distancia de 5.86 km, luego de lo cual se toma un camino de terracería rumbo a la costa por $1.66 \mathrm{~km}$.

Figura 2. Localización de los bancos de arena sílica (1845'35.86" N, 95²42'19.17" 0)

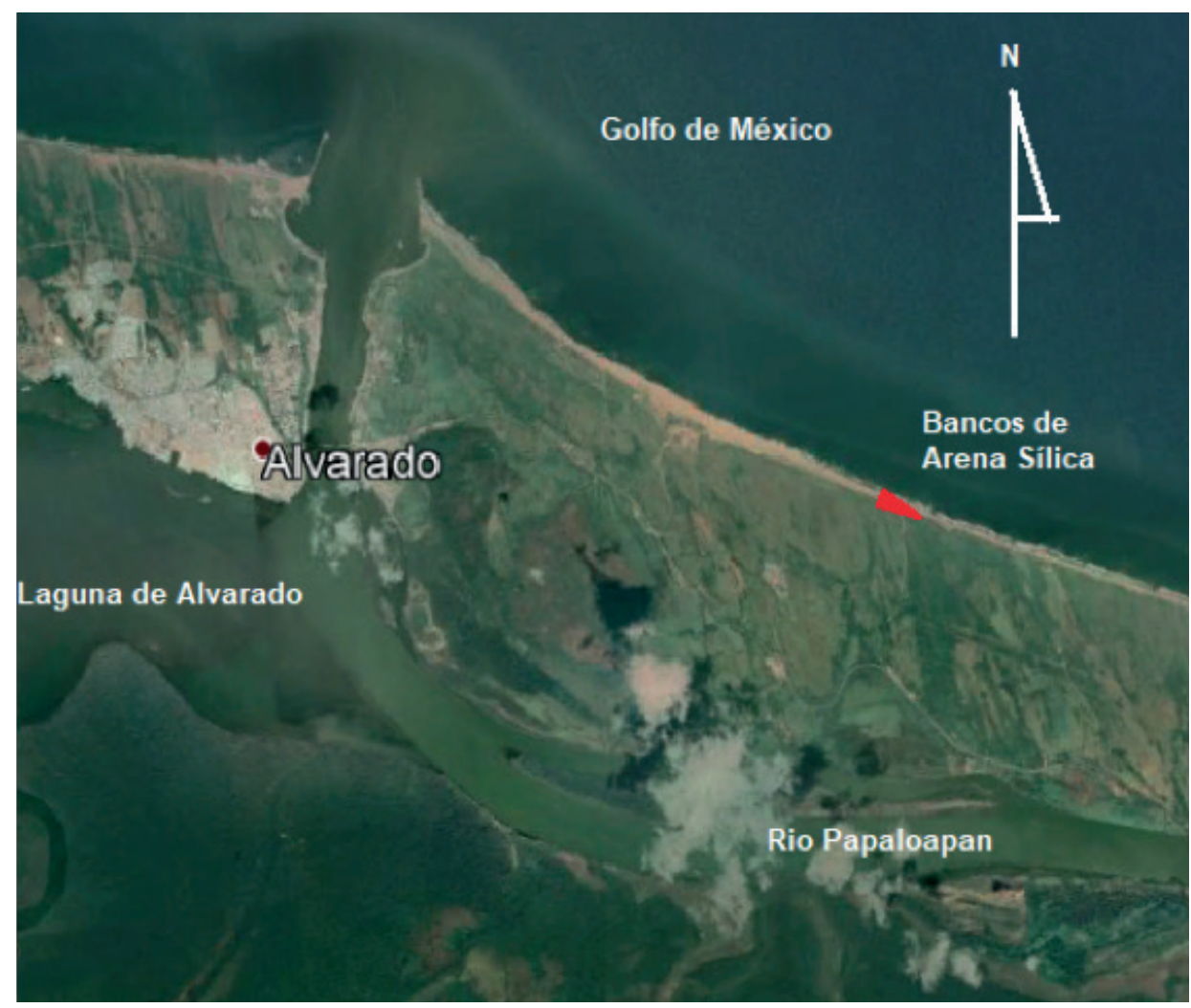

Fuente: Google Earth (2019) Modificada por Estévez

\section{c) Colecta y tratamiento de la arena sílica}

La arena sílica fue recolectada en el mes de julio del año 2020, del banco costero, por medio de maquinaria, propiedad de la empresa Sílices de México y llevada a sus instalaciones ubicadas en Km. 55 + 500 carretera Federal 180, tramo Alvarado-Lerdo, en la congregación de Chocotán, en el Municipio de Alvarado, Veracruz. En este lugar se procesan 25 toneladas diarias mediante el secado con un horno rotatorio, con quemador y atomizador (figura 3), que usa como combustible diésel, para que mediante la introducción de la arena por medio de una tolva, esta avance en contra flujo con la corriente de calor, que se genera por el quemador, y sea retirada la humedad presente. 
Figura 3. Horno de secado de sílices de México

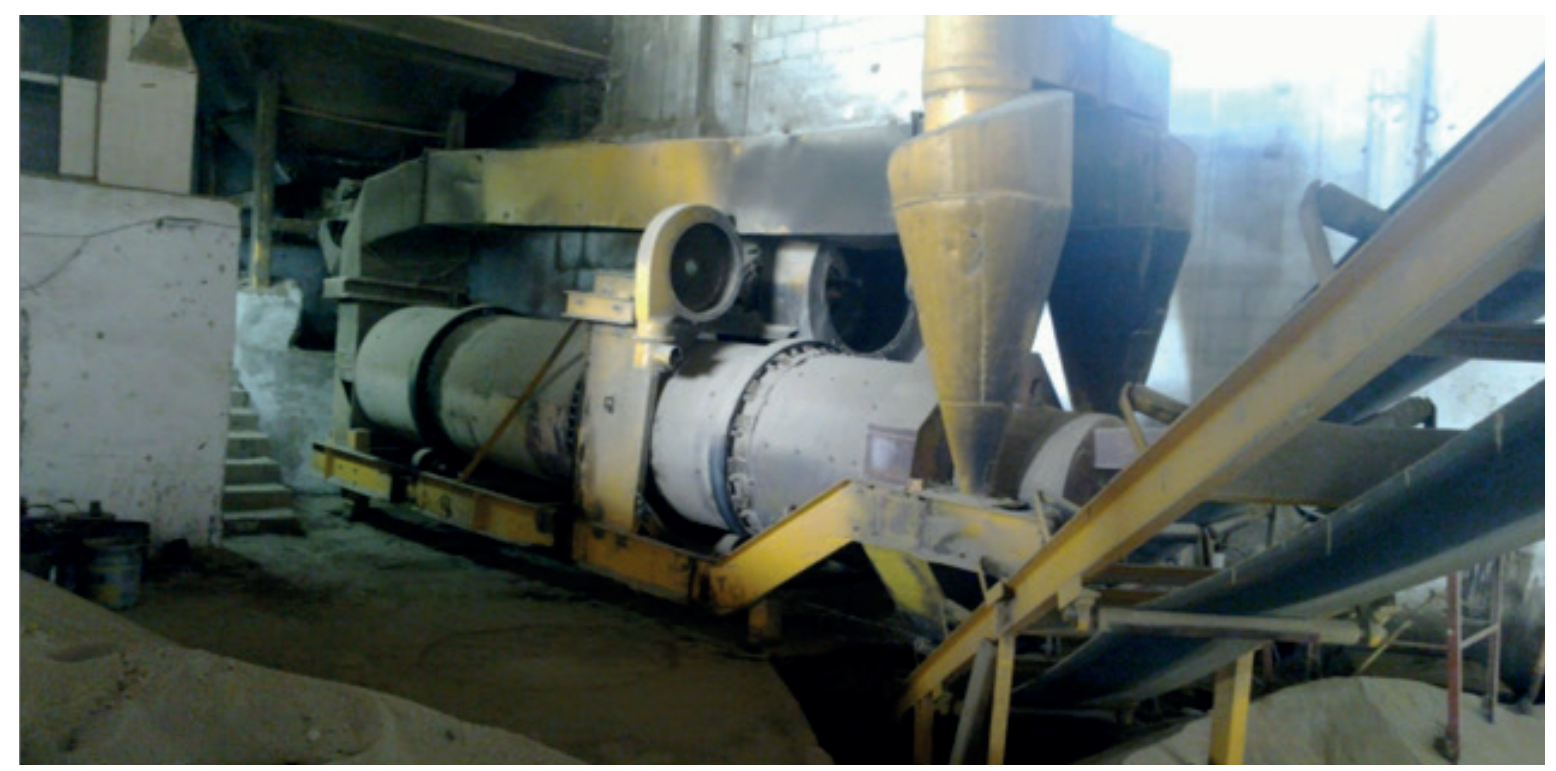

Fuente: Propia

La empresa sílices de México embala la arena sílica en sacos de $25 \mathrm{~kg}$, de los cuales se obtuvieron tres sacos de arena, procesada con un total de $75 \mathrm{~kg}$ para este análisis, los mismos que fueron trasladados por el propietario de la empresa a las instalaciones del Instituto Tecnológico de Veracruz, en donde se los identificaron como lotes 1, 2 y 3.

\section{d) Análisis granulométrico de la arena silica}

El análisis granulométrico de la arena sílica se realizó utilizando mallas de números 14, 25, 35, 45 y 60 en la escala de Tyler, mediante un tamizador de laboratorio de fisicoquímica, tomando muestras por triplicado de 1000 gramos de cada uno de los sacos proporcionados por la empresa.

\section{e) Contenido de humedad}

El contenido de humedad se determinó por triplicado para cada lote, con la utilización de una termobalanza, que es un analizador de humedad Adam modelo PMB, tomando muestras de 20 gramos, de cada uno de los tres lotes diferentes.

\section{f) Determinación de silicio y fierro en la arena sílica}

Para la determinación de silicio y fierro en la arena sílica, esta se trituró hasta malla 100 con un tamaño de partícula de $149 \mu \mathrm{m}$; se colocaron $0.002 \mathrm{~g}$ de la muestra y se trataron con $10 \mathrm{ml}$ de $\mathrm{HNO}_{3}$ al $10 \%$ y se diluyeron en un litro de agua destilada. Posteriormente se tomaron lecturas con un fotómetro Hanna HI83300, que utiliza un modo de medición de absorbancia que permite la utilización de los estándares CAL Check, para validar el rendimiento del sistema; este equipo permite seleccionar una de las cuatro longitudes de onda de la luz (420 nm, 525 nm, 575 nm y $610 \mathrm{~nm}$ ), que mide y traza su propia curva de concentración contra absorbancia. Todas las muestras se leyeron por triplicado. 
El método para la determinación de sílice se basa en la adaptación del estándar ASTM D859 - 16 por azul de heteropoliácido, de molibdeno, y el de fierro por modificación del método TPTZ (2,4,6-tripiridil-1,3,5-triazina).

\section{g) Análisis estadístico}

Para el análisis los datos obtenidos se aplicaron pruebas de análisis de medias de Tukey y Fisher con un nivel de confianza del $95 \%$, con el programa Minitab, a fin de encontrar el error estándar de la media tabular y las diferencias mínimas estadísticas, de los datos obtenidos en los resultados del laboratorio.

\section{Resultados}

Las principales propiedades físicas que se debe de tomar en cuenta para que un material pueda ser utilizado como barrera reactiva permeable es la granulometría de las partículas, la densidad y el contenido de humedad, ya que son esenciales para determinar la cantidad de relleno en la barrera reactiva permeable (Yin et al., 2017).

\section{TAMAÑO DE PARTÍCULAS}

El tamaño de las partículas es uno de los mecanismos clave para las BRP, ya que es un factor primordial para la adsorción de contaminantes en medios minerales (ITRC, 2011; Cucarella y Renman, 2009); además, los dímetros homogéneos permiten que la conductividad hidráulica (permeabilidad), de los medios reactivos, sea mayor que la del suelo circundante, permitiendo que el flujo de agua subterránea penetre fácilmente a través de la barrera reactiva permeable (Asokbunyarat y Annachhatre 2017).

El análisis granulométrico de la arena sílica se realizó utilizando mallas de números 14,25, 35, 45 y 60 en la escala de Tyler, mediante un tamizador de laboratorio en el Laboratorio de Fisicoquímica del Instituto Tecnológico de Veracruz, en mayo del 2019. Se realizaron tomando 1000 gramos de muestra de cada uno de los tres lotes diferentes, proporcionados por la empresa sílice de México, en saquillos de $50 \mathrm{~kg}$. Se operó el equipo por un periodo de cinco minutos y el procedimiento se repitió dos veces más, para obtener el resultado por triplicado (tabla 2).

Tabla 2. Análisis granulométrico de la arena sílica de Alvarado, Veracruz, México

\begin{tabular}{|c|c|c|c|c|c|c|c|c|c|}
\hline Malla & \multicolumn{3}{|c|}{$\begin{array}{c}\text { Lote 1 } \\
\text { Peso en g }\end{array}$} & \multicolumn{3}{c|}{$\begin{array}{c}\text { Lote 2 } \\
\text { Peso en g }\end{array}$} & \multicolumn{3}{c|}{$\begin{array}{c}\text { Lote 3 } \\
\text { Peso en g }\end{array}$} \\
\hline 14 & 0.0 & 0.2 & 0.0 & 0.0 & 0.0 & 0.0 & 0.1 & 0.0 & 0.0 \\
\hline 25 & 997.2 & 996.8 & 997.3 & 995.3 & 996.0 & 995.8 & 997.6 & 997.2 & 997.3 \\
\hline 35 & 1.4 & 1.6 & 1.4 & 1.4 & 1.5 & 1.7 & 1.5 & 1.4 & 1.4 \\
\hline 45 & 0.2 & 0.3 & 0.2 & 0.4 & 0.6 & 0.5 & 0.3 & 0.3 & 0.3 \\
\hline 60 & 0.2 & 0.2 & 0.2 & 0.3 & 0.6 & 0.4 & 0.3 & 0.3 & 0.2 \\
\hline Cernido & 1.0 & 0.9 & 0.9 & 2.6 & 1.3 & 1.6 & 0.2 & 0.8 & 0.8 \\
\hline
\end{tabular}

De acuerdo con los datos obtenidos, el promedio del peso de las muestras de malla 25, el $99.67 \%$ de las partículas obtenidas, tienen un tamaño de 0.707 mm, y los resultados del programa minitab, en cuanto al análisis de las medias, refiere que el tamaño no presenta estadísticamente variación para ninguno de los tres lotes. 
En cuanto a la malla 25, en ambos estadísticos el lote 1 y 3 no tienen diferencia estadística; sin embargo, el lote 2 sí presenta.

Figura 4. prueba de Fisher para malla 25

Figura 5. Prueba de Tukey para malla 25
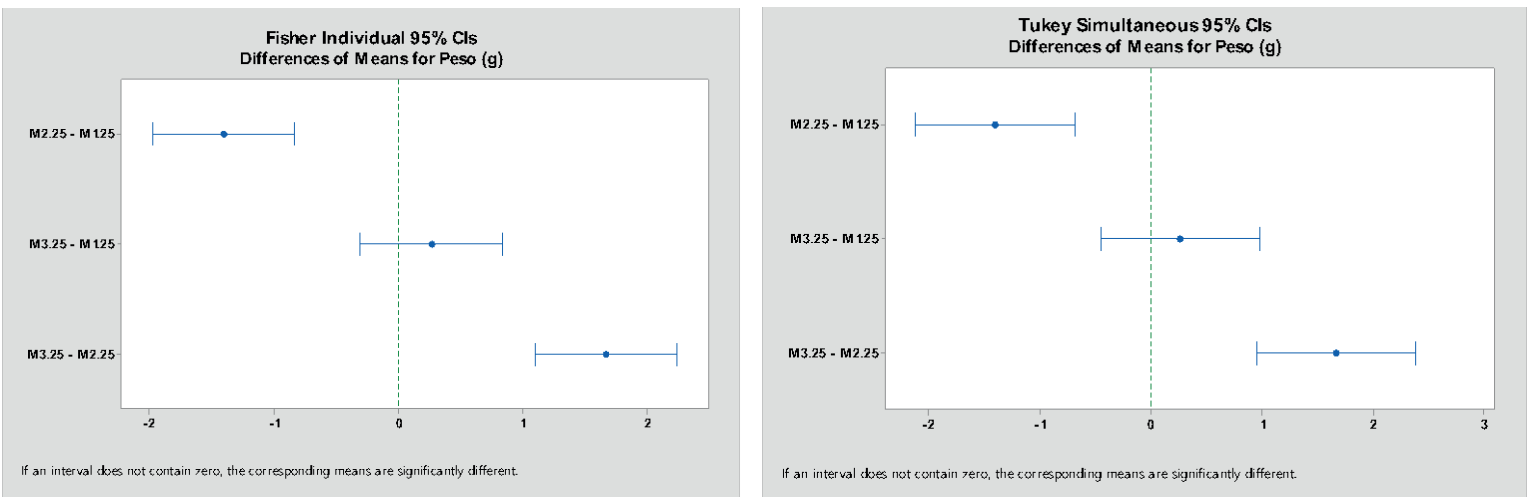

\section{CONTENIDO DE HUMEDAD}

El contenido de humedad en el material para las BRP es esencial para la conductividad hidráulica (Yin et al., 2017), ya que junto con el gradiente hidráulico determinan la velocidad y dirección del flujo de los contaminantes en el agua subterránea (ITRC, 2011).

Para el caso de la humedad, se colocaron tres muestras de entre 25 y 26 gramos de cada uno de los lotes diferentes y se procedió a determinar el porcentaje en masa de humedad; los resultados que se obtuvieron se reportan en la tabla 3.

Tabla 3. Humedad relativa de la arena sílica de Alvarado, Ver

\begin{tabular}{|c|c|c|c|c|c|c|c|c|}
\hline \multicolumn{3}{|c|}{$\begin{array}{c}\text { Lote 1 } \\
\text { \% Masa }\end{array}$} & \multicolumn{3}{c|}{$\begin{array}{c}\text { Lote 2 } \\
\% \text { Masa }\end{array}$} & \multicolumn{3}{c|}{ Lote 3 } \\
\% Masa \\
\hline 0.05 & 0.05 & 0.05 & 0.05 & 0.06 & 0.05 & 0.05 & 0.05 & 0.06 \\
\hline
\end{tabular}

La media aritmética del porcentaje de masa de las nueve muestras es de $0.052 \%$, y de acuerdo con las pruebas estadísticas de Tukey y Fisher, ninguno de los lotes tiene diferencia estadística significativas.

Figura 6. prueba de Fisher para humedad

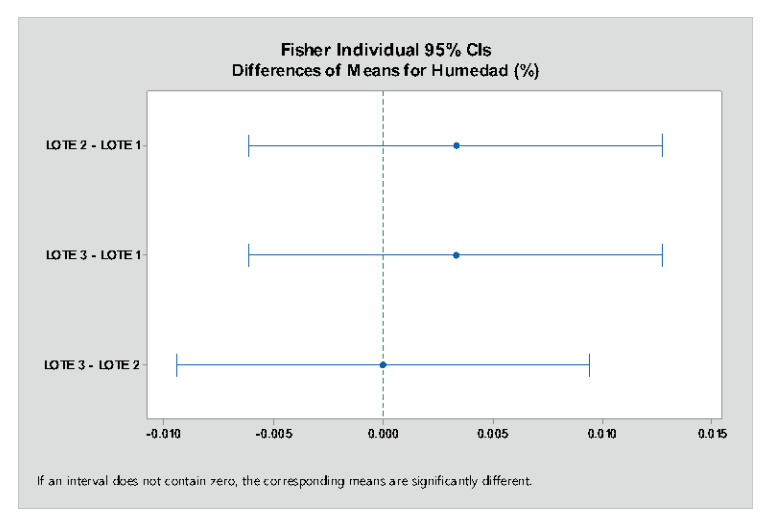

Figura 7. Prueba de Tukey para humedad

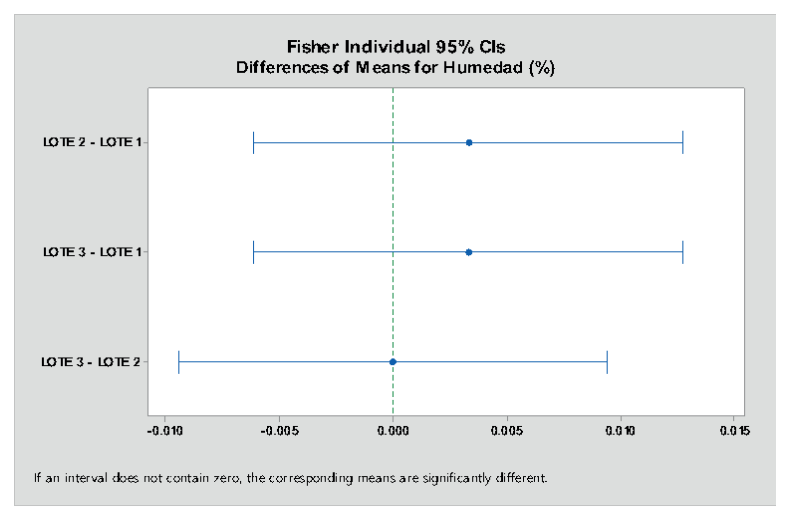




\section{ConTENIDO DE SÍLICE Y FIERRO}

Las muestras colectadas fueron tratadas con $\mathrm{HNO}_{3}$ al $10 \%$ y con abundante agua desionizada; posterior a lo cual se filtraron por medio de carbón activado, para eliminar los colores de interferencia. Las lecturas de los metales $\mathrm{Si}\left(\mathrm{SiO}_{2}\right)$, y Fe $\left(\mathrm{Fe}_{2} \mathrm{O}_{3}\right)$ se realizaron con un fotómetro Hanna HI83300, que utiliza un modo de medición de absorbancia, que permite la utilización de estándares, basados en la adaptación del método ASTM D859 - 16 por azul de heteropoliácido de molibdeno, y el de fierro por modificación del método TPTZ (2,4,6-tripiridil-1,3,5-triazina), a longitudes de onda de $610 \mathrm{~nm}$ para el silicio y de $420 \mathrm{~nm}$ para el hierro. Todas las muestras se leyeron por triplicado (tabla 4).

Tabla 4. Concentración de sílice y fierro en la arena sílica de Alvarado, Ver., México

\begin{tabular}{|c|c|c|c|c|c|}
\hline \multicolumn{2}{|c|}{ Lote 1 mg/l } & \multicolumn{2}{c|}{ Lote 2 mg/l } & \multicolumn{2}{c|}{ Lote 3 mg/l } \\
\hline $\mathrm{SiO}_{2}$ & $\mathrm{Fe}_{2} \mathrm{O}_{3}$ & $\mathrm{SiO}_{2}$ & $\mathrm{Fe}_{2} \mathrm{O}_{3}$ & $\mathrm{SiO}_{2}$ & $\mathrm{Fe}_{2} \mathrm{O}_{3}$ \\
\hline 1.74 & 0.05 & 1.80 & 0.05 & 1.74 & 0.06 \\
\hline 1.75 & 0.05 & 1.73 & 0.06 & 1.75 & 0.05 \\
\hline 1.73 & 0.05 & 1.74 & 0.06 & 1.75 & 0.06 \\
\hline
\end{tabular}

Considerando que se pesaron $0.002 \mathrm{~g}$ de muestras en balanza analítica, se puede determinar que el porcentaje de sílice y de hierro en la arena sílica, es el siguiente (tabla 5).

Tabla 5. Concentración de sílice y fierro (en porcentaje) en la arena sílica de Alvarado, Ver.

\begin{tabular}{|c|c|c|c|c|c|}
\hline \multicolumn{2}{|c|}{ Lote 1 } & \multicolumn{2}{c|}{ Lote 2 } & \multicolumn{2}{c|}{ Lote 3 } \\
\hline $\mathrm{SiO}_{2}$ & $\mathrm{Fe}_{2} \mathrm{O}_{3}$ & $\mathrm{SiO}_{2}$ & $\mathrm{Fe}_{2} \mathrm{O}_{3}$ & $\mathrm{SiO}_{2}$ & $\mathrm{Fe}_{2} \mathrm{O}_{3}$ \\
\hline 87.0 & 2.5 & 90.0 & 2.5 & 87.0 & 3.0 \\
\hline 87.5 & 2.5 & 86.5 & 3.0 & 87.5 & 2.5 \\
\hline 86.5 & 2.5 & 87.0 & 3.0 & 87.5 & 3.0 \\
\hline
\end{tabular}

Como se puede observar en la tabla 5, el valor medio de la concentración de $\mathrm{SiO}_{2}$ y $\mathrm{Fe}_{2} \mathrm{O}_{3}$ en la arena sílica de la costa del municipio de Alvarado, Veracruz, es de 87.38 \% y $2.72 \%$ respectivamente. Los resultados del programa Minitab, en cuanto al análisis de las medias, refiere que la concentración no presenta estadísticamente variación para ninguno de los tres lotes, ya que las pruebas de Tukey y Fisher para el $\mathrm{SiO}_{2}$ y el Fe $\mathrm{O}_{3}$ presentan distribuciones estadísticas homogéneas para ambos componentes.

Figura 8. prueba de Fisher para $\mathrm{SiO}_{2}$

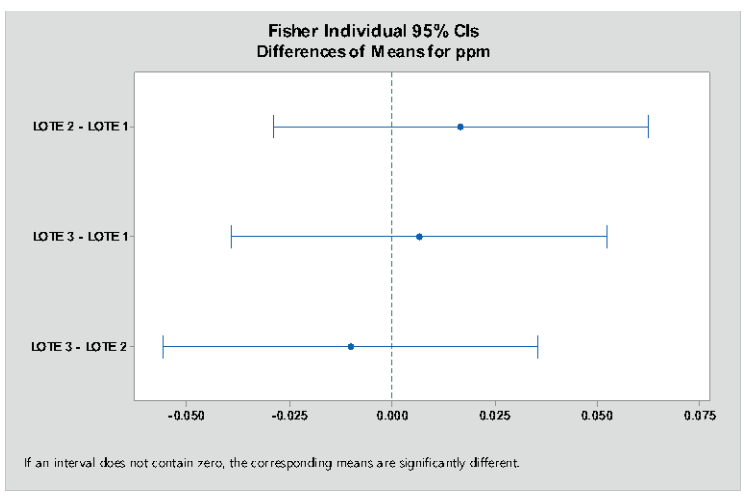

Figura 9. Prueba de Tukey $\mathrm{SiO}_{2}$

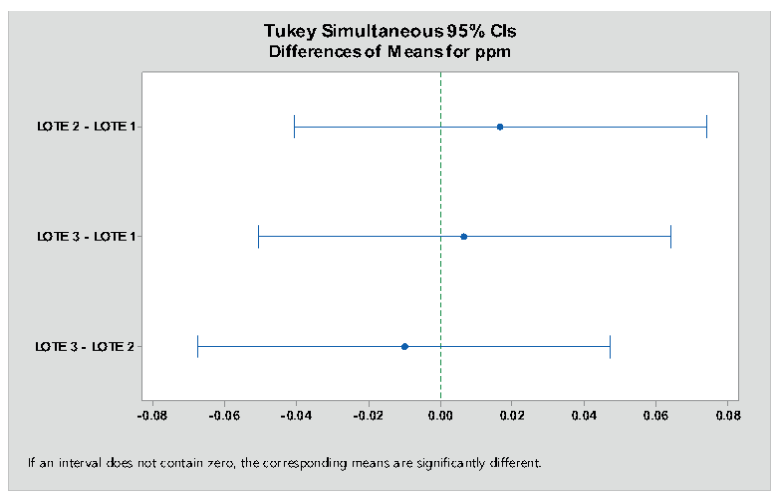


Figura 10. prueba de Fisher para $\mathrm{Fe}_{2} \mathrm{O}_{3}$

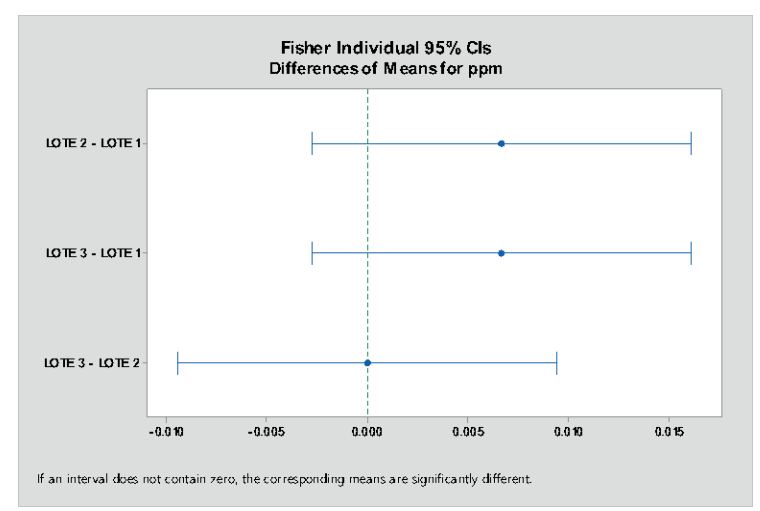

Figura 11. Prueba de Tukey $\mathrm{Fe}_{2} \mathrm{O}_{3}$

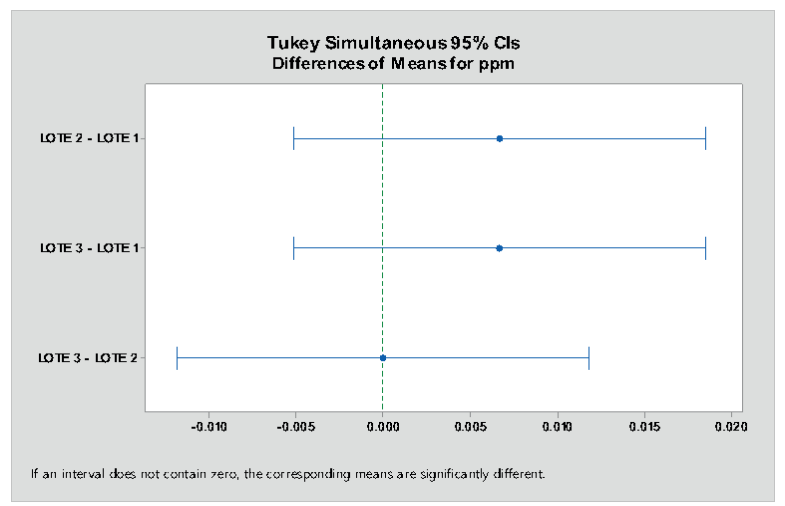

\section{Discusión}

Los medios reactivos utilizados en barreras permeables deben ser compatibles con el entorno subsuperficial (Faisal et al. 2018). En el caso de la arena sílica del Municipio de Alvarado, Veracruz, este material, que se obtiene de manera natural y que proviene de los sedimentos que arrastra el río Papalopan hacia el mar, donde por las corrientes marinas se deposita en la zona de estudio (Díaz et al., 2018), se ha usado como biofiltros, ya que se ha comprobado que tienen la capacidad de eliminar la turbidez, color, sólidos suspendidos, compuestos orgánicos e inorgánicos y bacterias (Payan, 2013).

El análisis granulométrico resultante (tabla 2) de la arena sílica de las costas de Alvarado, Veracruz, México, determinó que el 99.67 \% de las partículas tiene un tamaño de 0.707 mm. Esta homogeneidad en el tamaño de las partículas permitirá asegurar un flujo uniforme en las BRP (Arenas, 2017), y una conductividad hidráulica adecuada para la retención de iones metálicos divalentes (Courcelles et al., 2011), mediante el mecanismo de adsorción por el medio mineral (ITRC, 2011), lo que permitirá minimizar las limitaciones de flujo del agua de los acuíferos subterráneos, ya que por su tamaño homogéneo no se presentaría el bloqueo entre los espacios intergranulares (Faisal, 2018).

Los porcentajes de retención para $\mathrm{Pb}$ y Zn en columnas con material reactivo con tamaños de partículas de 2 a 3 mm, son muy elevados, cercanos al 99 \% y del 90 al 95 \% para Cd y As, disminuyendo ligeramente cuando aumenta la granulometría del adsorbente (Pérez, 2014). Bajo condiciones de homogeneidad granulométrica, las arenas sílicas de Fairmount Minerals and Subsidiaries-Best Sand, Ohio en los Estados Unidos, con tamaños de 0.2 a 0.5 mm, ha presentado eficiencias la remoción de $\mathrm{PO}_{4}^{3-}$ en rangos de 21 al 58\% (Agrawal et al., 2011).

La arena sílica de las costas del municipio de Alvarado, Veracruz, tiene una concentración media de $\mathrm{SiO}_{2}$ y $\mathrm{Fe}_{2} \mathrm{O}_{3}$ (tabla 5 ) de $87.38 \%$ y $2.72 \%$ respectivamente; estos valores son muy similares a la arena residual de fundición, que se ha utilizado como barrera reactiva permeable en aguas subterráneas ( $94.36 \%$ de $\mathrm{SiO}_{2}$ y $2.12 \%$ de $\mathrm{Fe}_{2} \mathrm{O}_{3}$ ), las cuales han reportado eficiencias para la remoción de cobre hasta 93 \% (Faisal y Ahmed, 2014).

El contenido de $\mathrm{SiO}_{2}$ se encuentra en los promedios obtenibles en bancos de materiales para diversos fines (Narasimha, 2016; Díaz, 2018), ya que el rango de valores oscila entre el 85 y el 90 \%; sin embargo, el contenido de $\mathrm{Fe}_{2} \mathrm{O}_{3}$ en comparación con otras zonas (Díaz, 2018), es superior a los materiales que se han utilizado para la inmovilización de metales pesados como 
cobre, níquel, cromo, plomo, mercurio, cadmio (Ademe, 2014). Si bien los silicatos pueden influir negativamente en las barreras reactivas permeables, diseñadas para la eliminación de metales traza (ITRC, 2011), el alto contenido de $\mathrm{Fe}^{3+}$ permite que se realicen los mecanismos de degradación química de metales pesados como el $\mathrm{Cr}^{6+}$ a $\mathrm{Cr}^{3+}$ (Chimenos y Navarro, 2002).

\section{Conclusiones y recomendaciones}

El material reactivo es el principal componente utilizado en el sistema de barreras reactivas permeables (BRP), para facilitar la eliminación de contaminantes de las aguas subterráneas. Los principales criterios para la selección del material son la disponibilidad del material, la reactividad, la estabilidad mecánica, la rentabilidad, la conductividad hidráulica, la compatibilidad ambiental y la seguridad para su uso (Roehl et al., 2005; ITRC 2011; Yin et al., 2017).

Considerando los criterios de selección del material para las barreras reactivas permeables, la arena sílica proveniente de las costas del municipio de Alvarado, Veracruz posee alta estabilidad, ya que por su alto contenido de $\mathrm{Fe}_{2} \mathrm{O}_{3}$ permanecerá activa durante un período de tiempo más largo. La cantidad y disponibilidad permiten que sea transportada con costos accesibles para la mayor parte del país, y dado que su dímetro es homogéneo a 0.707 mm, permitirá que su permeabilidad sea mayor a la de los suelos convencionales, además de ser un material compatible con el ambiente y seguro para su trabajo.

Los medios reactivos utilizados en barreras permeables deben ser compatibles con el entorno subsuperficial. Es decir, los medios no deben causar reacciones químicas adversas o productos, al reaccionar con los componentes en la columna de contaminantes, y no deben actuar como una posible fuente de contaminantes. El material debe permitir el flujo de agua subterránea, lo cual se consigue con un tamaño de partículas que no sea excesivamente pequeño y con una granulometría heterogénea, para que no resulten bloqueados los espacios intergranulares (Maitra, 2019; Mital et al. 2020).

El silicio, como el de la arena sílica del municipio de Alvarado, Veracruz, cuenta con una estructura a través de la cual los cationes intercambiables son adsorbidos, ya que actúa como medio poroso y cuando es permeado por agua, los metales que pasan a través de este material se atrapan vía intercambio iónico, permitiendo que este sistema de retención (Mohan y Gandhimathi, 2009) tenga una disponibilidad muy alta, pues estos bancos son utilizado0s para material de Sand-Blast y se distribuye a todo el país.

Mediante el uso de la arena sílica de la costa del municipio de Alvarado, Veracruz, como barrera reactiva permeable, se puede lograr una atenuación más rápida de los contaminantes del agua subterránea en condiciones controladas. La barrera también evita que los contaminantes provenientes de los lixiviados de los basureros no controlados en el agua subterránea migren a acuíferos no contaminados (Chimenos y Navarro, 2002; Cordeiro y Viera, 2020).

La arena sílica posee alta estabilidad por su alto contenido de $\mathrm{Fe}_{2} \mathrm{O}_{3^{\prime}}$ y es un material que se tiene en cantidad y disponibilidad para la exigencia de remediación de acuíferos contaminados con lixiviados de basureros no controlados, ya que puede ser transportado con costos accesibles para la mayor parte del país por las compañías ubicadas en la zona de Alvarado, Veracruz y, dado que su dímetro es homogéneo, permitirá una mayor permeabilidad sin que se degrade el material, el cual es compatible con el ambiente porque solo es extraído, tamizado y secado. De igual manera, como se muestra en las comparaciones realizadas en el presente artículo, sus características físico-químicas son estadísticamente similares, lo cual garantiza resultados de calidad en sus aplicaciones. 
Las barreras reactivas permeables carecen de partes móviles, equipamiento y ruido. Los materiales reactivos se colocan por medio de zanjas, lo que no perjudica a las aguas subterráneas ni a las personas y como el proceso de limpieza es bajo tierra, no se tiene contacto con los contaminantes. (Arenas, 2017; Mital et al., 2020).

Se recomienda, como siguiente paso, determinar la eficiencia de remoción de metales pesados y de carga orgánica, para poder realizar la ingeniería necesaria para la remoción de contaminantes de los acuíferos subterráneos.

\section{Referencias}

ADEME. (2014). Barrières réactives. Cahier SKB, 7761. https://bit.ly/3IR1dJ0

Arenas, G. (2017). Barreras reactivas aplicables en acuifferos para la remoción de materia orgánica proveniente de residuos urbanos (tesis de maestría). Programa de Maestría y Doctorado en Ingeniería (Ingeniería Ambiental - Agua). Universidad Nacional Autónoma de México.

Agrawal, S.; King, K.; Fischer, R.; Woner, D. (2011). P043- Removal by and Permeability of Industrial Byproducts and Minerals: Granulated Blast Furnace Slag, Cement Kiln Dust, Coconut Shell Activated Carbon, Silica Sand, and Zeolite. Water Air Soil Pollut 219, 91-101.

Arenas-Moreno, G. (2017). Barreras reactivas aplicables en acuíferos para la remoción de materia orgánica proveniente de residuos urbanos. [Tesis de Maestría, Ingeniería Ambiental-Agua, UNAM, México]

Asokbunyarat,V.; Lens, P.; Annachhatre, A. (2017). Permeable Reactive Barriers for Heavy Metal Removal, Sustainable Heavy Metal Remediation. Vol. 1. Principles and Processes (pp. 65-100).

Bohn, H. L.; McNeal, B. L.; O'Connor, G. A. (1985). Soil Chemistry. USA: John Wiley \& Sons, Inc.

Bernaché, G. (2012). Riesgo de contaminación por disposición final de residuos. Un estudio de la región centro occidente de México. Rev. Int. Contam. Ambie 28, Sup. (1), 97-105.

Chimenos, J.; Navarro, A. (2002). Descontaminación de aguas subterráneas mediante barreras reactivas permeables. Tecnología del Agua 1, 38-45.

Cordeiro Andrade, D.; Vieira dos Santos, E. (2020). Combination of Electrokinetic Remediation with Permeable Reactive Barriers to Remove Organic Compounds from Soils. Current Opinion in Electrochemistry 22, 136-144. https://doi.org/10.1016/j.coelec.2020.06.002

Courcelles, B.; Modaressi-Farahmand-Razavi, A.; Gouvenot, D.; Esnault-Filet, A. (2011). Influence of Precipitates on Hydraulic Performance of Permeable Reactive Barrier Filter. Vol. 1. International Journal of Geomechanics, No. 2, April.

Cucarella, V.; Renman, G. (2009). Phosphorus Sorption Capacity of Filter Materials Used for On-site Wastewater Treatment Determined in Batch Experiments-A Comparative Study. J. Environ. Qual, (38), 381-392.

Díaz, M.; Campos, R.; Zamora, S.; Salgado, R. (2018). Cuantificación experimental del contenido masivo de $\mathrm{SiO}_{2}$ en depósitos eólicos de arena en una región de la cuenca del río Papaloapan, Veracruz, México. Vol. 22. Ingeniería Revista Académica de la Facultad de Ingeniería, Universidad Autónoma de Yucatán, 22, No. 1.

Dirección General de Desarrollo Minero. (2011). Perfil del mercado del sílice, Secretaría de Economía, Gobierno de México.

Faisal A., Ahmed M. (2014). Removal of Copper lons from Contaminated Groundwater Using Waste Foundry Sand as Permeable Reactive Barrier. Int. J. Environ. Sci. Technol, (12), 2613-2622.

Faisal, A.; Sulaymon, A.; Khaliefa, Q. (2018). A review of Permeable Reactive Barrier as Passive Sustainable Technology for Groundwater Remediation. Int. J. Environ. Sci. Technol, (15), 1123-1138.

Han, Z.; Ma, H.; Shi, G, et al. (2016). A review of Groundwater Contamination Near Municipal Solid Waste Landfill Sites in China. Sci. Total Environ, 569-570; 1255-1264. 
ITRC. (2011). Permeable Reactive Barrier: Technology Update. BRP-5. Washington, D.C.: Interstate Technology \& Regulatory Council, BRP: Technology Update Team. https://bit.ly/3jN2z5L

Liu, S.; Li, X.; Wang, H. (2011). Hydraulics Analysis for Groundwater Flow Through Permeable Reactive Barriers, Environ Model Assess (2011), 16:591-598.

Maitra S. (2019). Permeable Reactive Barrier: A Technology for Groundwater Remediation - A Mini Review. Research Journal of Life 5(1):203.

Miao, L.; Yang, G.; Tao, T.; Peng, Y. (2019). Recent Advances in Nitrogen Removal from Landfill Leachate Using Biological Treatments - A Review. J. Environ. Manag, (235),178-185.

Mittal, A.; Singh, R.; Chakma, S.; Gaurav, G. (2020). Permeable Reactive Barrier Technology for the Remediation of Groundwater Contaminated with Nitrate and Phosphate Resulted from Pit-Toilet Leachate. Vol. 37. Journal of Water Process Engineering.

Mohan, S.; Gandhimathi, R. (2009). Removal of Heavy Metal Ions from Municipal Solid Waste Leachate Using Coal Fly Ash as an Adsorbent. J Hazard Mater 169(1-3):351-359.

McRae, C. (1999). Evaluation of Reactive Materials for In Situ Treatment of Arsenic III, Arsenic V and Selenium VI Using Permeable Reactive Barriers: Laboratory Study, (MSc Thesis). University of Waterloo, Waterloo, Ontario, Canada.

Narasimha, l; Bubu, J. (2016). Investigations on Physical and Chemical Properties of High Silica Sand, Fe-Cr Slag and Blast Furnace Slag for Foundry Applications. Procedia Environmental Sciences (35), $583-596$.

NOM-083-Semarnat-083. (20 de octubre de 2004). Especificaciones de protección ambiental para la selección del sitio, diseño, construcción, operación, monitoreo, clausura y obras complementarias de un sitio de disposición final de residuos sólidos urbanos y de manejo especial. Diario Oficial de la Federación. México.

Naveen, B. P.; Mahapatra, D. M.; Sitharam, T. G.; Sivapullaiah, P. V., Ramachandra, T. V. (2017). Physico-chemical and Biological Characterization of Urban Municipal Landfill 373 Leachate. Environ. Pollut 220, pp. 1-12.

Payan, S. (2013). Estudio y diseño de biofiltro a partir de materia orgánica para el tratamiento de agua [Tesis de Doctorado, Centro de Investigación en Materiales Avanzados S.C.]. https://bit.ly/35c00tj

Pérez Espinoza, V. (2014). Inmovilización de elementos potencialmente tóxicos en zonas mineras abandonadas mediante la construcción de tecnosoles y barreras reactivas permeables [Tesis de Doctorado, Universidad de Murcia, España] https://bit.ly/3h5qHPb

Roehl, K. E.; Czurda, K.; Meggyes, T.; Simon, F. G.; Stewart, D. I. (2005). Permeable Reactive Barriers. In: Roehl KE, Meggyes T, Simon FG, Stewart DI (eds.) Long-term Performance of Permeable Reactive Barriers. Elsevier, Amsterdam Sciences, Bioinformatics, Pharmaceutical and Chemical Sciences 5(1) 203-217.

Scherer, M. M.; Richter, S.; Valentine, R. L.; Álvarez P. J. J. (2000). Chemistry and microbiology of permeable reactive barriers for in situ groundwater clean-up. Critical Reviews. Environmental Science and Technology 30(3): 363-411.

SEFIPLAN. (2012). Estudios regionales para la planeación. Información básica. Gobierno de Veracruz. https://bit.ly/3jM057D

Segura, R.; Martínez, G.; Apolinar, J.; García, M. (2015). Remoción de Cr6+ de lixiviados de tiraderos de basura con arcillas modificadas o activadas. Ciencia UANL 75, año 18, pp. 54-70.

Striegel, J.; Sanders, D. A.; Veenstra, J. (2001). Treatment of Contaminated Groundwater Using Permeable Reactive Barriers. Environmental Geosciences 8(4), 258-265.

Susunaga-Miranda, M. A.; Estévez-Garrido, B. M. (2018). Metales pesados en los lixiviados provenientes del basurero no controlado de la Ciudad de Veracruz. Vol. 5 Revista Iberoamericana de Ciencias 6 , 164-171.

Thiruvenkatachari, R.; Vigneswaran, S.; Naidu, R. (2008). Permeable Reactive Barrier for Groundwater Remediation. J Ind Eng Chem 14(2):145-156. 
Teta, C. \& Hikwa, T. (2017). Heavy Metal Contamination of Ground Water from an Unlined Landfill in Bulawayo, Zimbabwe. Journal of health \& pollution 7(15), 18-27.

Vázquez-Lule, A.; Rodríguez-Zúñiga, M.; Ramírez-García, P. (2009). Caracterización del sitio de manglar sistema lagunar de Alvarado Veracruz, en Comisión Nacional para el Conocimiento y Uso de la Biodiversidad (Conabio). Sitios de manglar con relevancia biológica y con necesidades de rehabilitación ecológica. Conabio, México, D.F

Vukojević, N.; Daković, A.; Ugrina, M. et al. (2017). Evaluation of Low-cost Sorbent as Potental Materials for In-Situ Remediation of Water Contaminated with Heavy Metals. Technologica Acta 10(2) 9-13.

Yin, S.; Herath, G.; Heng, S.; Kalpage, S. (2017). Using Permeable Reactive Barriers to Remediate Heavy Metal-Contaminated Groundwater through a Laboratory Column. Experiment American Journal of Environmental Sciences 13(2): 103-115 